\title{
Cathepsin-k expression in pulmonary lymphangioleiomyomatosis
}

\author{
Marco Chilosi ${ }^{1}$, Maurizio Pea ${ }^{2}$, Guido Martignoni ${ }^{1}$, Matteo Brunelli ${ }^{1}$, Stefano Gobbo ${ }^{1}$, \\ Venerino Poletti ${ }^{3}$ and Franco Bonetti ${ }^{1}$ \\ ${ }^{1}$ Department of Pathology, University of Verona, Verona, Italy; ${ }^{2}$ Anatomia Patologica, Ospedale Orlandi, \\ Bussolengo, Italy and ${ }^{3}$ Department of Diseases of the Thorax, GB Morgagni Hospital, Forlí, Italy
}

\begin{abstract}
Lymphangioleiomyomatosis is a rare and progressive lung cystic disease, caused by the infiltration of lung parenchyma by mesenchymal cells characterized by co-expression of contractile proteins and melanocytic markers. The pathogenesis of lymphangioleiomyomatosis is determined by mutations affecting tuberous sclerosis complex (TSC) genes, with eventual deregulation of the Rheb/mTOR/p70S6K pathway, and the potential therapeutic activity of mTOR inhibitors is currently under investigation. To better understand the molecular mechanisms involved in the pathogenesis of lymphangioleiomyomatosis, we investigated the expression of cathepsin-k (a papain-like cysteine protease with high matrix-degrading activity). The rationale of this choice was based on the recent demonstration that mTOR inhibitors can regulate major functional activities of osteoclasts, including the expression of cathepsin-k. The immunohistochemical study included 12 cases of lymphangioleiomyomatosis. Twelve angiomyolipomas and several lung diseases (sarcoidosis, organizing pneumonia, usual interstitial pneumonia, emphysema) were investigated as controls. In all lymphangioleiomyomatosis cases, strong cathepsin-k immunoreactivity was demonstrated, restricted to lymphangioleiomyomatosis cells. Similar expression levels were observed in renal angiomyolipomas. These observations extend the knowledge regarding the immunophenotypic profile of lymphangioleiomyomatosis cells, and provide a useful new marker for diagnosis in difficult cases (eg, in small transbronchial biopsies). The strong expression of such a potent papain-like cysteine protease in lymphangioleiomyomatosis cells can significantly contribute to the progressive remodelling of lung parenchyma observed in this deadly disease, with eventual formation of lung cysts. It is possible to speculate that mTOR inhibitors may exert part of their action by limiting the destructive remodelling of lung structure.
\end{abstract}

Modern Pathology (2009) 22, 161-166; doi:10.1038/modpathol.2008.189; published online 5 December 2008

Keywords: lymphangioleiomyomatosis; cathepsin-k; angiomyolipoma; mTOR; immunohistochemistry; lung remodelling

Lymphangioleiomyomatosis is a rare and progressive cystic disease that affects the lungs of women, usually in premenopausal age, associated with mutations in tuberous sclerosis genes and renal angiomyolipomas. ${ }^{1-6}$ The abnormalities affecting the lung parenchyma are caused by progressive infiltration of perilymphatic spaces by peculiar smoothmuscle cells co-expressing contractile proteins (mainly $\alpha$-smooth-muscle actin and desmin) and melanocytic markers such as HMB45, HMSA-1, MelanA/Mart1 and microphtalmia transcription factor (MITF) ${ }^{7-9}$ The nature and normal counterpart of lymphangioleiomyomatosis cells is not known,

Correspondence: Professor M Chilosi, MD, Department of Pathology, University of Verona, Strada Le Grazie, no. 8, Verona 37134, Italy.

E-mail: marco.chilosi@univr.it

Received 26 June 2008; revised and accepted 29 August 2008; published online 5 December 2008 although it is possible to speculate that they derive from mesenchymal precursors modified by definite genetic abnormalities inducing a neoplastic phenotype. ${ }^{10,11}$ These features taken together, lymphangioleiomyomatosis can be considered as part of the spectrum of proliferative lesions that have been previously defined by our group under the name of 'perivascular epithelioid cell tumours' (PEComas), including angiomyolipoma, clear-cell 'sugar' tumour of the lung and extrapulmonary sites and rare clear-cell tumours at other anatomical sites. ${ }^{2,12-14}$ This class of tumours is characterized by distinctive morphologic, immunohistochemical, ultrastructural and genetic features and are defined by the World Health Organization as 'mesenchymal tumours composed of histologically and immunohistochemically distinctive perivascular epithelioid cells'.2 Although the inclusion of pulmonary lymphangioleiomyomatosis within this group of lesions has been questioned, lymphangioleiomyomatosis shares 

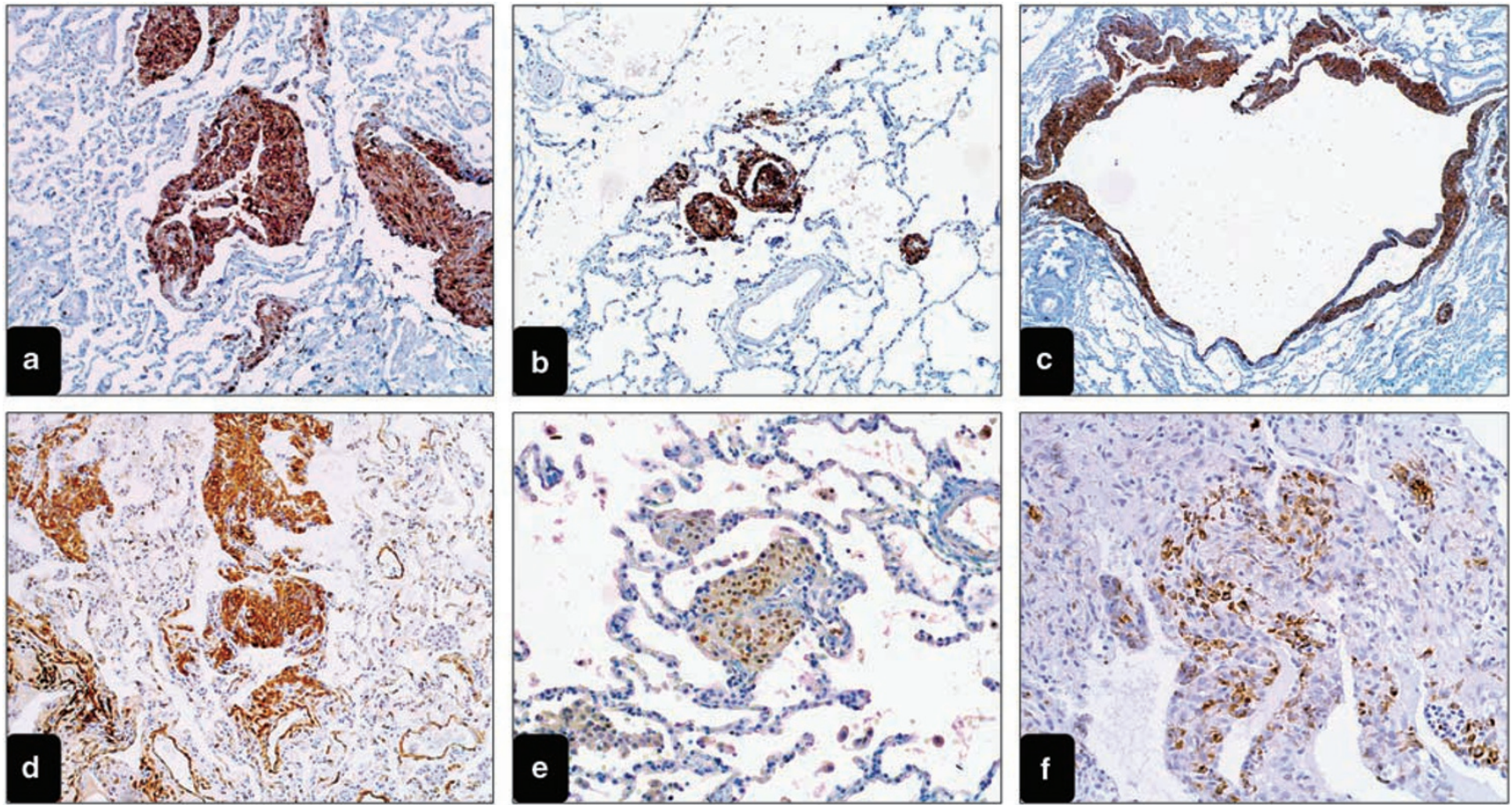

Figure 1 Immunohistochemical demonstration of cathepsin-k expression in pulmonary lymphangioleiomyomatosis (a-c). Lymphangioleiomyomatosis cells in both nodular and cystic lesions exhibit strong and homogeneous immunostaining. Lymphangioleiomyomatosis cells co-express smooth-muscle (d, $\alpha$-SMA), and melanocytic (e, MITF; f, HMB45) markers.

with PEComas most immunophenotypic features, and typically show the genetic alterations of the tuberous sclerosis complex (TSC), an autosomal dominant genetic disease because of either losses of TSC1 (9q34) or TSC2 (16p13.3) genes. ${ }^{15,16}$

In recent years, great advances have been made in understanding the molecular pathogenesis of TSC and related lesions. In particular, the critical role of TSC genes products has been revealed in the regulation of the Rheb/mTOR/p70S6K pathway. ${ }^{17}$ Recently, Kenerson et $a l^{18}$ demonstrated TSC1/2 inactivation and $\mathrm{m}$-TOR hyperactivation in nonTSC angiomyolipomas, and in extra-renal PEComas using immunohistochemistry and western blot analysis. Immunohistochemical evaluation of downstream products of the mTOR pathway can provide useful diagnostic markers in this context, and PEComas do express in fact abnormal levels of p70S6K, a protein kinase activated by mTOR, and also predictive information regarding the potential therapeutic use of molecules related to rapamycin that are currently under evaluation for lymphangioleiomyomatosis and PEComas. ${ }^{19-23}$

The mTOR/S6K pathway is involved in different cell systems, and in particular in the stimulation of cell survival and functions of osteoclasts. ${ }^{24}$ Major functional activity of osteoclasts is related to their strong expression of cathepsin-k, a papain-like cysteine protease, ${ }^{25}$ with high matrix (collagen, elastin)-degrading activity. ${ }^{26,27}$ Interestingly, the expression of cathepsin-k in osteoclasts is regulated by $\mathrm{MITF}^{28}$ and is significantly reduced by mTOR inhibitors. $^{29}$ MITF and cathepsin-k are also expressed in melanocytes and melanomas, ${ }^{30}$ where mTOR is also activated and critical for the regulation of apoptosis. ${ }^{31,32}$ All these data taken together, it is possible to hypothesize that MITF and cathepsin$\mathrm{k}$ are in fact under the control of mTOR pathway, and could serve as further molecular markers for evaluating mTOR pathway activation in lymphangioleiomyomatosis. As there are no data regarding cathepsin-k expression in lymphangioleiomyomatosis currently available, we investigated in this study the expression of cathepsin-k in lymphangioleiomyomatosis and angiomyolipomas by using immunohistochemistry.

\section{Materials and methods}

\section{Study Population}

We investigated 12 cases of pulmonary lymphangioleiomyomatosis retrieved from the file of the Department of Pathology of the Verona University, together with 12 cases of renal angiomyolipomas. The diagnosis of lymphangioleiomyomatosis was based on clinical, radiological and histological features, according to updated criteria. ${ }^{2,6}$ Histology and immunohistochemistry demonstrated the typical features of lymphangioleiomyomatosis, with aggregates of spindle- and epithelioid-shaped cells co-expressing smooth-muscle and melanocytic 
markers bordering cystic spaces and forming irregular perilymphatic nodules (Figure 1). Control cases included normal lung tissue samples (3 cases), sarcoidosis (3 cases), organizing pneumonia (3 samples), usual interstitial pneumonia (3 cases), pulmonary emphysema (3 cases) and normal pulmonary parenchyma from tissue adjacent to lung tumours (10 cases).

\section{Immunohistochemical Staining and Antibodies}

All tissue samples were formalin-fixed, paraffinembedded according to standard methods. Sections from tissue blocks of lymphangioleiomyomatosis, angiomyolipomas and control cases were immunostained with the following antibodies: $\alpha$-smoothmuscle actin ( $\alpha$-SMA-clone 2A4, Dako, Glonstrup, Denmark), HMB45 (Dako); MITF (clone D5, Neomarkers/LabVision, Fremont, CA), MART1/MELANA (clone A103, Biogenex, San Ramon, CA), and two different mouse monoclonal antibodies recognizing human cathepsin-k (clone CK4, Novocastra, Newcastle, UK and clone 3F9, Abcam, Cambridge, UK), using previously described methods. ${ }^{33}$ Heatinduced antigen retrieval for cathepsin-k was performed using a microwave oven and $0.01 \mathrm{~mol} / \mathrm{l}$ of citrate buffer, $\mathrm{pH}$ 8.0, for $30 \mathrm{~min}$. All samples were processed using a sensitive 'Bond polymer Refine' detection system in an automated Bond immunostainer (Vision-Biosystem, Menarini, Florence, Italy). Sections incubated without the primary antibody served as a negative control.

\section{Results}

\section{Lymphangioleiomyomatosis Samples}

In all cases a strong immunoreactivity in spindleand epithelioid-shaped cells was demonstrated using both cathepsin-k-specific antibodies with comparable intensities. Immunoreactivity for cathepsin-k was definitely higher than that observed using HMB45 and MITF, and clearly depicted cystic spaces and nodules in lymphangioleiomyomatosis samples.

Smooth-muscle cells of vascular and bronchiolar walls were completely negative for cathepsin-k, and also negative were the macrophages observed within alveolar spaces, thus clearly highlighting the foci of lymphangioleiomyomatosis occurring within the lung parenchyma. We observed co-expression of $\alpha$-SMA, HMB45, MITF and MART1/MELAN-A with cathepsin-k in cells composing the foci of lymphangioleiomyomatosis (Figure 1).

\section{Angiomyolipomas Samples}

In all cases of renal angiomyolipoma strong cathepsin-k expression was demonstrated, clearly distinguishing the neoplastic lesion from the adjacent normal renal parenchyma (Figure 2). Both cathepsin-k-specific antibodies showed comparable intensities. All types of PEC cells including spindle, epithelioid smooth-muscle and adipocyte-like cells were strongly and homogeneously immunoreactive for cathepsin-k, this confirming the phenotypic identity of this morphologically heterogeneous cell group. ${ }^{2}$ The endothelium and the wall of the vessels present in the tumours were unstained with cathepsin-k. $\alpha$-SMA, HMB45, MITF and MART1/ MELAN-A were regularly expressed in angiomyolipomas as previously described. ${ }^{2,22}$

\section{Control Samples}

Among the different cases of lung disease investigated as control, the only samples containing cathepsin-k expression were the three sarcoidosis cases where strong immunoreactivity was confined to epithelioid macrophages and giant cells in granulomas. In usual interstitial pneumonia and organizing pneumonia samples, strong expression was demonstrated in myofibroblast collections (fibroblastic foci of usual interstitial pneumonia and inflammatory polyps in organizing pneumonia samples) as previously described. ${ }^{34}$ No cathepsin-k immunoexpression was observed in 3 pulmonary emphysema from lung biopsies and from 10 normal pulmonary parenchyma adjacent to lung tumours.

\section{Discussion}

In this paper, we first demonstrate that the spindleand epithelioid-shaped cells of lymphangioleyomiomatosis exhibit strong expression of cathepsin-k, and that similar strong expression is observed in neoplastic cells in all cases of renal angiomyolipoma. These observations extend the knowledge regarding the immunophenotypic profile of lymphangioleiomyomatosis and PEC cells, and provide a useful new marker for diagnosis of lymphangioleiomyomatosis in difficult cases (eg, in small transbronchial biopsies). ${ }^{35,36}$ The co-expression of $\alpha$-SMA and melanocyte markers (HMB45, MART1) is currently used as a specific immunophenotypic profile to distinguish and characterize the foci of pulmonary lymphangioleiomyomatosis on tissue sections, but in some cases these melanocyte markers are only expressed at low levels, with occasional negative results, thus limiting the sensitivity of the analysis. According to our results, cathepsin-k is a very robust marker in this context, with immunoreactivity comparable to $\alpha$-SMA in all cases (Figure 1). In addition, the specificity of cathepsin-k as a lymphangioleiomyomatosis marker is very high, as all other cell types expressing $\alpha$-smooth-muscle cells within the pulmonary tissue are completely negative for cathepsin-k, including the walls of blood vessels and airways. The only cells expressing comparable levels of cathepsin-k in 

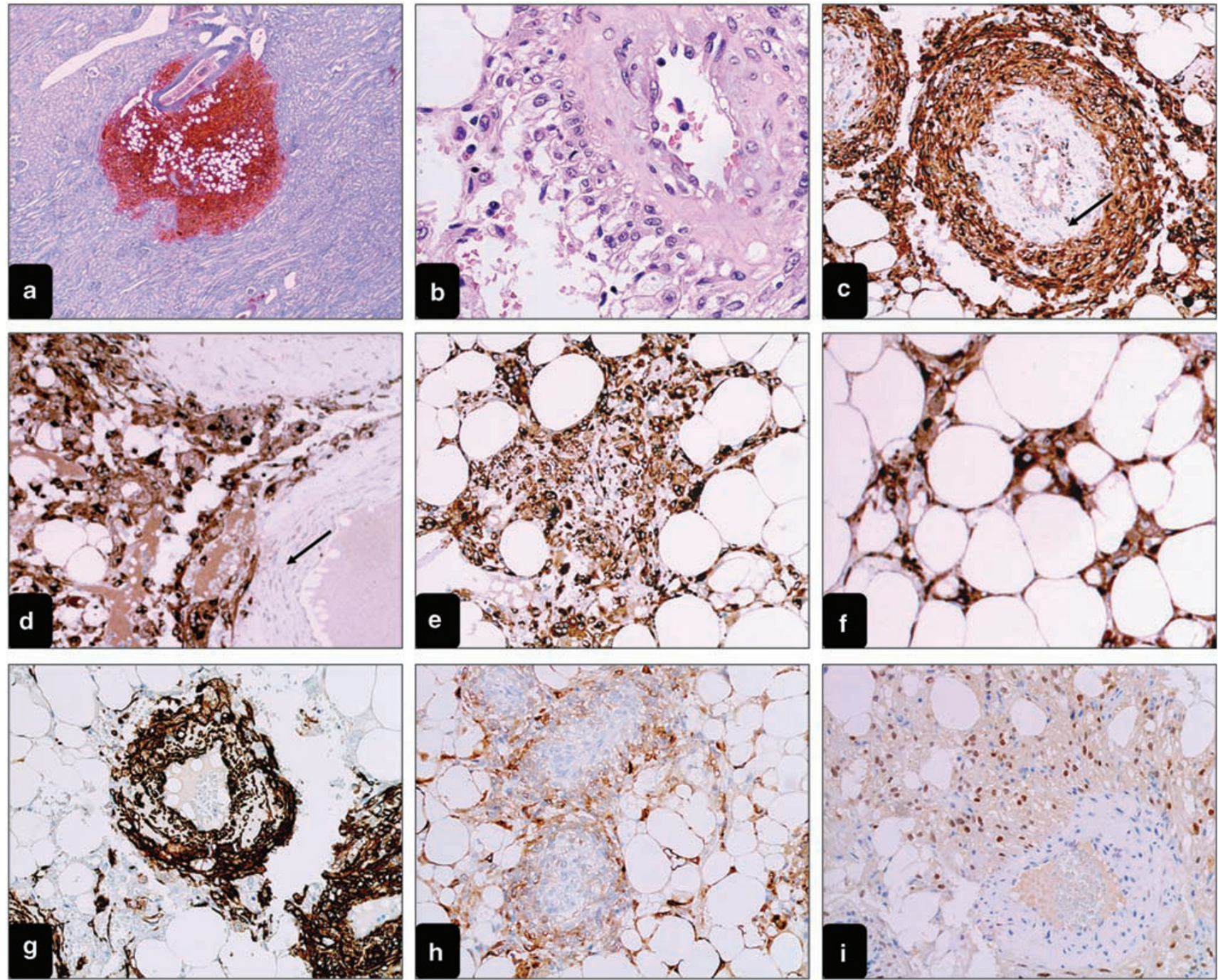

Figure 2 Immunohistochemical demonstration of strong cathepsin-k expression in renal angiomyolipoma and no immunoexpression in normal renal parenchyma harbouring the lesion (a). All types of PEC cells including spindle and epithelioid smooth-muscle cells (b, $\mathrm{H} \& \mathrm{E}$ ) and adipocyte-like cells are strongly and homogeneously immunostain by cathepsin-k (c-f). PEC cells (b, H\&E) co-express smoothmuscle (g, $\alpha$-SMA), and melanocytic antigens (h, MART1; i, MITF) features. Vascular structures (c, d) did not show immunoexpression (arrows).

control lung samples were granulomas observed in sarcoidosis, but these cells could not represent diagnostic problems because of their distinct morphologic and other immunophenotypic features. Myofibroblasts in fibroblast foci of usual interstitial pneumonia and organizing pneumonia samples express discrete levels of cathepsin-k, as previously described, ${ }^{34}$ but these cells can be easily distinguished by morphology and immunophenotype.

As far as the pathogenesis of lymphangioleiomyomatosis is concerned, the demonstration of strong cathepsin-k expression in lymphangioleiomyomatosis can contribute to explain some features of the disease, and in particular the typical formation of lung cysts. Cathepsin-k is in fact a papain-like cysteine protease with high matrix-degrading activity on collagen and elastin, and this activity can significantly contribute, together with other previously described proteases (metalloproteinases) to the progressive structural damage and remodelling of pulmonary parenchyma. ${ }^{37,38}$ Cathepsin-k is in fact unique among proteases in degrading a wide range of collagens, at variance with matrix metalloproteinases that are able to cleave triple helical collagen only at specific single sites. ${ }^{39}$

The constant finding of cathepsin-k immunoexpression in the spindle- and epithelioid smoothmuscle and adipocyte-like cells (PECs) in renal angiomyolipomas confirms the phenotypic identity of these morphologically heterogeneous cells and their relationship with the cellular elements composing the pulmonary lymphangioleyomiomatosis of the lung. Therefore, this marker should add to the immunophenotypical profile, including $\alpha$-SMA, 
HMB-45, MART1 and Melan-A markers, which characterizes the PEC cells composing both renal angiomyolipoma and pulmonary lymphangioleyomiomatosis.

Finally, it is possible to speculate that mTOR inhibitors recently proposed as a new therapeutic option for lymphangioleiomyomatosis, can exert part of their action by limiting the expression of cathepsin-k, thus contrasting the destructive remodelling on lung structure. In favour to this interesting possibility is the observation that rapamycin and its analogues have efficacy in limiting the bone resorption mediated by osteoclastic cathepsin-k, ${ }^{29}$ and can interfere with the proliferation of melanoma, a tumour exhibiting mTOR pathway activation, as well as MITF and cathepsin-k expression. ${ }^{31,30,32}$ Finally, it is worth noting that abnormal bone mineral density has been demonstrated at high prevalence in lymphangioleiomyomatosis. ${ }^{40}$ Although oestrogen deficiency has been hypothesized as a possible cause for the observed bone loss, ${ }^{40}$ the possible influence of cathepsin-k overproduction, and/or the derangement of mTOR pathway in osteoclasts in lymphangioleiomyomatosis patients warrants further consideration in future investigations.

\section{Acknowledgement}

This study was supported by the European Union FP7 Health Research Grant number HEALTH-F42008-202047.

\section{References}

1 Crooks DM, Pacheco-Rodriguez G, DeCastro RM, et al. Molecular and genetic analysis of disseminated neoplastic cells in lymphangioleiomyomatosis. Proc Natl Acad Sci USA 2004;101:17462-17467.

2 Martignoni G, Pea M, Reghellin D, et al. PEComas: the past, the present and the future. Virchows Arch 2008;452:119-132.

3 Ryu JH, Moss J, Beck GJ, et al. The NHLBI lymphangioleiomyomatosis registry: characteristics of 230 patients at enrollment. Am J Respir Crit Care Med 2006;173:105-111.

4 Smolarek TA, Wessner LL, McCormack FX, et al. Evidence that lymphangiomyomatosis is caused by TSC2 mutations: chromosome 16p13 loss of heterozygosity in angiomyolipomas and lymph nodes from women with lymphangiomyomatosis. Am J Hum Genet 1998;62:810-815.

5 Steagall WK, Taveira-DaSilva AM, Moss J. Clinical and molecular insights into lymphangioleiomyomatosis. Sarcoidosis Vasc Diffuse Lung Dis 2005;22:S49-S66.

6 Taveira-DaSilva AM, Steagall WK, Moss J. Lymphangioleiomyomatosis. Cancer Control 2006;13:276-285.

7 Bonetti F, Pea M, Martignoni G, et al. Cellular heterogeneity in lymphangiomyomatosis of the lung. Hum Pathol 1991;22:727-728.

8 Matsumoto Y, Horiba K, Usuki J, et al. Markers of cell proliferation and expression of melanosomal antigen in lymphangioleiomyomatosis. Am J Respir Cell Mol Biol 1999;21:327-336.

9 Zhe X, Schuger L. Combined smooth muscle and melanocytic differentiation in lymphangioleiomyomatosis. J Histochem Cytochem 2004;52:1537-1542.

10 Krymskaya VP. Smooth muscle-like cells in pulmonary lymphangioleiomyomatosis. Proc Am Thorac Soc 2008;5:119-126.

11 Pacheco-Rodriguez G, Steagall WK, Crooks DM, et al. TSC2 loss in lymphangioleiomyomatosis cells correlated with expression of CD44v6, a molecular determinant of metastasis. Cancer Res 2007;67: 10573-10581.

12 Bonetti F, Pea M, Martignoni G, et al. Clear cell ('sugar') tumor of the lung is a lesion strictly related to angiomyolipoma-the concept of a family of lesions characterized by the presence of the perivascular epithelioid cells (PEC). Pathology 1994;26:230-236.

13 Pea M, Bonetti F, Zamboni G, et al. Melanocyte-marker HMB-45 is regularly expressed in angiomyolipoma of the kidney. Pathology 1991;23:185-188.

14 Pea M, Martignoni G, Zamboni G, et al. Perivascular epithelioid cell. Am J Surg Pathol 1996;20:1149-1153.

15 European Chromosome 16 Tuberous Sclerosis Consortium. Identification and characterization of the tuberous sclerosis gene on chromosome 16. Cell 1993;75:1305-1315.

16 van Slegtenhorst $\mathrm{M}$, de Hoogt R, Hermans $\mathrm{C}$, et al. Identification of the tuberous sclerosis gene TSC1 on chromosome 9q34. Science 1997;277:805-808.

17 Kwiatkowski DJ. Tuberous sclerosis: from tubers to mTOR. Ann Hum Genet 2003;67:87-96.

18 Kenerson H, Folpe AL, Takayama TK, et al. Activation of the mTOR pathway in sporadic angiomyolipomas and other perivascular epithelioid cell neoplasms. Hum Pathol 2007;38:1361-1371.

19 Bissler JJ, McCormack FX, Young LR, et al. Sirolimus for angiomyolipoma in tuberous sclerosis complex or lymphangioleiomyomatosis. N Engl J Med 2008;358:140-151.

20 Davies DM, Johnson SR, Tattersfield AE, et al. Sirolimus therapy in tuberous sclerosis or sporadic lymphangioleiomyomatosis. N Engl J Med 2008;358:200-203.

21 Morton JM, McLean C, Booth SS, et al. Regression of pulmonary lymphangioleiomyomatosis associated retroperitoneal angiomyolipoma post-lung transplantation with rapamycin treatment. J Heart Lung Transplant 2008;27:462-465.

22 Paul E, Thiele E. Efficacy of sirolimus in treating tuberous sclerosis and lymphangioleiomyomatosis. N Engl J Med 2008;358:190-192.

23 Taillé C, Debray MP, Crestani B. Sirolimus treatment for pulmonary lymphangioleiomyomatosis. Ann Intern Med 2007;146:687-688.

24 Glantschnig H, Fisher JE, Wesolowski G, et al. M-CSF, TNFalpha and RANK ligand promote osteoclast survival by signaling through mTOR/S6 kinase. Cell Death Differ 2003;10:1165-1177.

25 Brömme D, Okamoto K, Wang BB, et al. Human cathepsin $\mathrm{O} 2$, a matrix protein-degrading cysteine protease expressed in osteoclasts. Functional expression of human cathepsin $\mathrm{O} 2$ in Spodoptera frugiperda and characterization of the enzyme. J Biol Chem 1996;271:2126-2132.

26 Drake FH, Dodds RA, James IE, et al. Cathepsin K, but not cathepsin B, L, or S, is abundantly expressed in human osteoclasts. J Biol Chem 1996;271:12511-12516. 
27 Inaoka T, Bilbe G, Ishibashi O, et al. Molecular cloning of human cDNA for cathepsin K: novel cysteine proteinase predominantly expressed in bone. Biochem Biophys Res Commun 1995;206:89-96.

28 Motyckova G, Weilbaecher KN, Horstmann M, et al. Linking osteopetrosis and pycnodysostosis: regulation of cathepsin $\mathrm{K}$ expression by the microphthalmia transcription factor family. Proc Natl Acad Sci USA 2001;98:5798-5803.

29 Kneissel M, Luong-Nguyen NH, Baptist $\mathrm{M}$, et al. Everolimus suppresses cancellous bone loss, bone resorption, and cathepsin $\mathrm{K}$ expression by osteoclasts. Bone 2004;35:1144-1156.

30 Karbowniczek M, Spittle CS, Morrison T, et al. mTOR is activated in the majority of malignant melanomas. J Invest Dermatol 2008;128:980-987.

31 Bundscherer A, Hafner C, Maisch T, et al. Antiproliferative and proapoptotic effects of rapamycin and celecoxib in malignant melanoma cell lines. Oncol Rep 2008;19:547-553.

32 Quintanilla-Dieck MJ, Codriansky K, Keady M, et al. Cathepsin $\mathrm{K}$ in melanoma invasion. J Invest Dermatol 2008;128:2281-2288.

33 Chilosi M, Poletti V, Zamò A, et al. Aberrant Wnt/betacatenin pathway activation in idiopathic pulmonary fibrosis. Am J Pathol 2003;162:1495-1502.
34 Bühling F, Röcken C, Brasch F, et al. Pivotal role of cathepsin $\mathrm{K}$ in lung fibrosis. Am J Pathol 2004;164:2203-2216.

35 Bonetti F, Chiodera PL, Pea M, et al. Transbronchial biopsy in lymphangiomyomatosis of the lung. HMB45 for diagnosis. Am J Surg Pathol 1993;17:1092-1102.

36 Poletti V, Chilosi M, Olivieri D. Diagnostic invasive procedures in diffuse infiltrative lung diseases. Respiration 2004;71:107-119.

37 Hayashi T, Fleming MV, Stetler-Stevenson WG, et al. Immunohistochemical study of matrix metalloproteinases (MMPs) and their tissue inhibitors (TIMPs) in pulmonary lymphangioleiomyomatosis (LAM). Hum Pathol 1997;28:1071-1078.

38 Merrilees MJ, Hankin EJ, Black JL, et al. Matrix proteoglycans and remodelling of interstitial lung tissue in lymphangioleiomyomatosis. J Pathol 2004;203:653-660.

39 Garnero P, Borel O, Byrjalsen I, et al. The collagenolytic activity of cathepsin $\mathrm{K}$ is unique among mammalian proteases. J Biol Chem 1996;273: 32347-32352.

40 Taveira-Dasilva AM, Stylianou MP, Hedin CJ, et al. Bone mineral density in lymphangioleiomyomatosis. Am J Respir Crit Care Med 2005;171:61-67. 